\title{
Juvenile Nasopharyngeal Angiofibroma in a 16 Year-Old Male: Diagnosis and Surgical Treatment
}

\author{
Johanna Choremis ${ }^{\dagger}$ and Saul Frenkiel* ${ }^{*}$ M.D., F.R.C.P.(C.)
}

\section{INTRODUCTION}

Juvenile nasopharyngeal angiofibroma (JNA), as its name suggests, is a combined vascular and fibrous neoplasm arising in the nasopharynx of prepubertal and adolescent males (ages 7 to 19 years, mean age 14 years) $(1,2)$. The tumor exhibits a strong tendency to bleed (3) and, despite being microscopically benign, frequently exhibits destructive and aggressive behavior (4). JNA usually presents as a nasal obstruction with intermittent, spontaneous epistaxis.

\section{THE CASE}

A 16-year-old Caucasian male presented at the emergency room with severe, spontaneous, right-sided epistaxis. Before determining the cause of the nosebleed, the patient required urgent posterior and anterior packing with balloon catheters to control the bleeding. The patient had no history of trauma; instead, the epistaxis was spontaneous, severe, and accompanied by nasal congestion. The packs were removed two days later and examination of the nasal chambers revealed a right-sided fleshy red mass in the region of the posterior nasal chamber. This observation narrowed the differential diagnosis to a tumor (benign or malignant) or arteriovenous malformation (5).

A computerized tomography (CT) scan was ordered to confirm the mass and to determine its extent. The scan showed a nasopharyngeal mass extending superiorly towards the sphenoid sinus, growing into both posterior choanae; it did not extend intracranially or into either left

\footnotetext{
* To whom correspondence should be addressed: Chief, Department of Otolaryngology, Jewish General Hospital, Room E-209, 3755 Côte Ste-Catherine, Montreal, Quebec, H3T 1E2

$\dagger$ Faculty of Medicine, McGill University, Montreal, Quebec, Canada
}

or right pterygopalatine fossa (Figure 1). Minimal bony erosion and remodeling were noted at the level of the skull base, suggesting that the lesion was a slow growing tumor. Magnetic resonance imaging (MRI) further characterized the lesion to be an enhancing mass within the nasopharynx (Figure 2). Given these findings, the differential diagnosis included rhabdomyosarcoma (a malignant neoplasm of skeletal muscle origin), lymphoma (a malignant neoplasm of lymphoid or reticuloendothelial origin) or angiofibroma.

Considering the patient's age and presentation with epistaxis, there was a strong suspicion of JNA, but a biopsy was needed to make a final diagnosis. Using an endoscopic approach, the biopsy was performed and the bleeding that ensued was readily controlled with pressure packing. On examination, the lesion had a fleshy appearance and histology indicated thin walled blood vessels and moderately cellular fibrous stroma (Figure 3), which is consistent with JNA (6).

A surgical procedure to remove the lesion was planned. However, further observations of the tumor, as well as occlusion of the feeding vessels, were needed. An arteriogram with subtraction technique indicated that the tumor was fed by a branch of the internal maxillary artery and a smaller feeding vessel from the internal carotid system. Embolization of the feeding vessels from the external carotid system using micron contour emboli was performed, without incident, in order to reduce bleeding at the time of operation. Figure 4 shows the arteriogram before (Figure 4A) and after (Figure 4B) embolization of the feeding vessels. The definitive surgical procedure was performed later the same day.

A transpalatal approach was used to expose the lesion in the nasopharynx. This was accomplished by an 


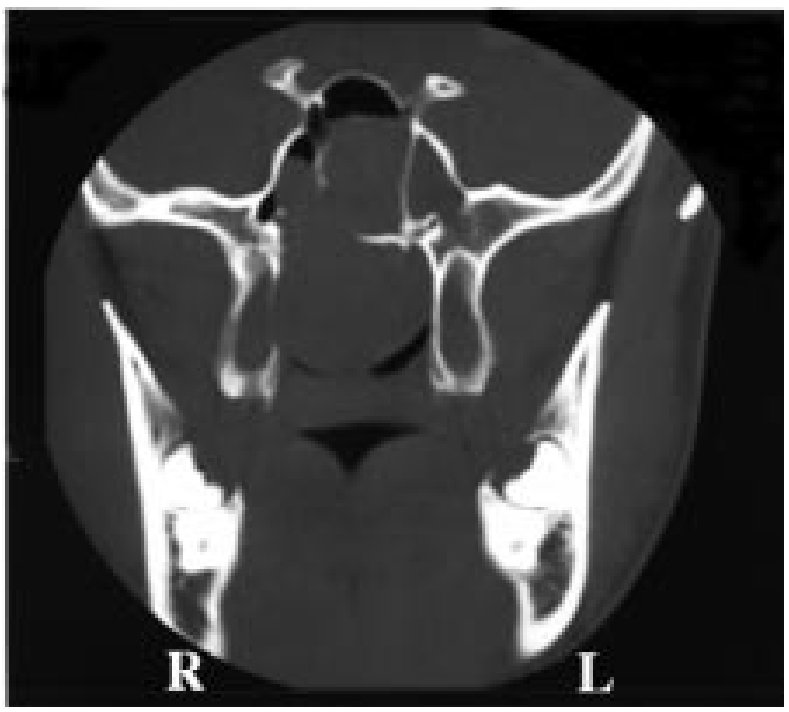

Figure 1. CT scan (coronal view) reveals a large nasopharyngeal mass extending superiorly towards the sphenoid sinus, consistent with the diagnosis of juvenile nasopharyngeal angiofibroma.

S-shaped incision through the soft palate splitting the free edge of the soft palate to the right of the hanging uvula. It was not necessary to remove any of the posterior edge of the hard palate, since a $30^{\circ}$ angled microscope was used to better visualize the skull base and to guide the surgery. The tumor was noted to fill the entire nasopharynx, abutting against each eustachian tube and projecting superiorly into an eroded anterior wall of sphenoid sinus. It did not, however, enter the pterygopalatine fossa on either side. The lesion was very firm and gritty, and was easily dissected en-block. Once removed, the specimen was noted to have a bilobed appearance with an indentation superiorly in the area where it abutted the roof of the sphenoid sinus in the region of the projecting pituitary gland where there was an intact bony wall. Bleeding was readily controlled with electrocauterization and pressure packing. Nevertheless, the total measured blood loss amounted to $1200 \mathrm{ml}$. A posterior nasal pressure pack was left in place for a period of five days, after which it was removed without complication.

The patient's recovery was uneventful and he was discharged from the hospital five days after his operation, in good condition. A follow-up exam two months after surgery indicated a well-healed nasopharynx without any evidence of residual tumor; monthly exams by laryngoscopy have shown no recurrence eight months post-surgery.

\section{DISCUSSION}

JNA is most commonly manifested in prepubertal and adolescent males (ages 7 to 19 years, mean age 14 years) $(1,2)$. Although it has been reported in females

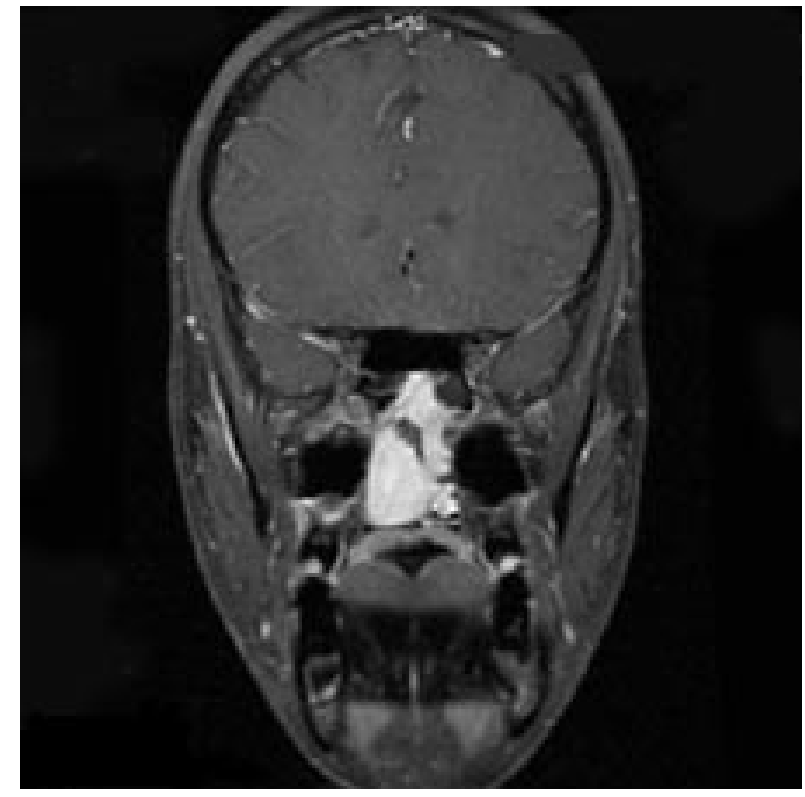

Figure 2. MRI scan (T1 weighted; coronal view) shows an enhancing mass in the nasopharynx.

and older males, this is extremely rare, and such cases should be closely scrutinized. JNA accounts for less than $0.05 \%$ of all benign lesions that originate in the nasopharynx. Despite this low incidence, JNA is the most common benign tumor originating in the nasopharynx of young males $(7,8)$.

As in the patient described, the clinical presentation of JNA usually consists of nasal obstruction and intermittent epistaxis. It is important to mention that the bleeding is unrelated to trauma and is completely spontaneous. Epistaxis can vary from occasional bleeding to severe hemorrhage that can cause chronic anemia in many patients (3).

In patients with JNA, anterior rhinoscopy will show abundant mucopurulent secretions as well as bowing of the nasal septum to the tumor free side. Posterior rhinoscopy will reveal the actual tumor, a pink or red mass, filling the nasopharynx. If it is extensive and large, the nasal bones are often splayed out and temple and cheek swelling may occur. If spread to the infratemporal fossa is considerable, trismus (lock-jaw), parotid gland bulging, and even proptosis (abnormal protrusion of the eye; only in the case of orbital fissure penetration), may occur. Failing vision is rare, but can occur with substantial extrapharyngeal extension, causing tenting of the optic nerve (3). Additionally, there can be stasis of secretions, sepsis, anosmia (inability to smell) or hyposmia due to blockage, as well as a nasal intonation or a plummy quality of voice if the soft palate is impinged upon. Deafness and otalgia may also occur if the eustachian tube is blocked. Headaches can occur due to chronic sinusitis resulting from obstructions (3). 


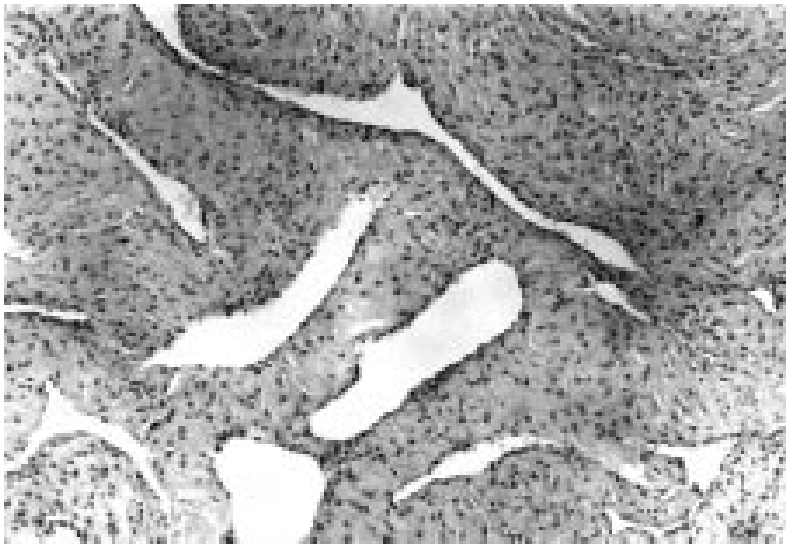

Figure 3. The tumor biopsy sample illustrates the thin walled blood vessels and moderately cellular fibrous stroma that are typical of juvenile nasopharyngeal angiofibroma.

In the diagnosis of JNA, plain films are of little value. They are not accurate and it is easy to mistake an opacity in the maxillary sinus or a soft tissue shadow in the postnasal space, for an antrochoanal polyp, among other things. CT with contrast localizes the mass and shows bony destruction and invasion of the skull base (3). If the tumor invades the pterygopalatine fossa, an axial view will show characteristic anterior bowing of the posterior wall of the maxillary sinus (9). MRI is superior for soft tissues, and an axial T1 weighted film will show typical salt and pepper appearance of JNA (10).

Selective arteriography with subtraction techniques will show a diagnostic vascular blush in the postnasal space and adjacent area (3). This imaging method will show the size and site of the lesion as well as the size and location of the feeding vessels. Figure 4 offers a good example of such a study. In Figure 4A, the contrasting agent lights up the tumor revealing that it is located in the nasopharynx and supplied by a branch of the internal maxillary artery and a smaller feeding vessel from the internal carotid system. The tumor is very well visualized with such a technique and along with other views, the surgeon can clearly appreciate the extent of the tumor. Lesions that can be radiographically misdiagnosed as a JNA tumor include angiomatous polyp, antrochoanal polyp, large adenoids, chordoma, lymphoma, rhabdomyosarcoma, melanoma, papilloma, and pyogenic granuloma. The reason many of these aforementioned diagnoses can resemble JNA radiographically is because they can all present as fleshy lesions that are often well vascularized.

If the diagnosis is in doubt, a transnasal biopsy may be performed (11). However, since the JNA lesion is extremely vascular, the nasal chamber may need to be immediately packed off to minimize blood loss, as was done in the current case. JNA is characterized by a firm, slightly spongy, lobulated tumor. Its nodularity increases with age and it is found to be whorled or spongy when sectioned. Mucous covered areas are usually seen as pink (nasopharyngeal area), whereas extrapharyngeal areas are white or grey. There is no true capsule, but the tumor is well demarcated from surrounding tissues (3). Microscopically, many vascular spaces of varying shape and size are seen in a stroma of fibrous tissue (Figure 3) (3). Smaller vascular channels lack smooth muscle which gives the tumor its high potential to bleed (12). The proportion of vascular spaces to fibrous tissue decreases with the age of the tumor, making the bleeding potential during resection unpredictable (3). Considering the patient did not complain of nosebleeds prior to this incident, it was very difficult to determine the age of the tumor. However, the CT scan did suggest it was slow growing.

Various treatment modalities are currently available for JNA, including surgical resection, embolization, external beam radiation, hormonal therapy, and combinations thereof. Studies indicate that surgery is a good form of treatment, especially when there is no intracranial involvement. The surgical approach depends on the pre-operative findings. A transpalatal approach is warranted for tumors confined to the nasopharynx (13), and was the approach used in the current case. A gingivobuccal incision can be added for access to the pterygomaxillary region (14). For tumors involving the nasal fossa and the pterygopalatine fossa, a lateral rhinotomy with resection of the medial antral wall is appropriate (3). When the infratemporal fossa is involved, a transnasal/transantral approach can be used $(15,16)$.

The recurrence rates of JNA range from 5\% to $11 \%$, post surgery. One study reported 18 cases treated surgically; 17 cases were resected through a transpalatal approach, and one was resected by a combined transpalatal, lateral rhinotomy, and transnasal approach (17). After a follow up period ranging from three months to nine years, all patients were alive, and only two recurrences requiring another procedure were reported. As well, no major complications were seen. The authors concluded that surgery should be the treatment of choice for tumors that have no intracranial involvement. A similar study reported 18 cases treated surgically, eight of which were also embolized preoperatively (18). Only one case extended intracranially, and this was the only case that recurred after a follow up of an average of six years, four months (range: 6 months to 17 years). Complications to consider with surgery include bleeding, palatal fistulas (especially with a transpalatal route right above the junction of the hard and soft palate), velopharyngeal incompetence, neural damage, and cerebrospinal fluid leaks. None of these 

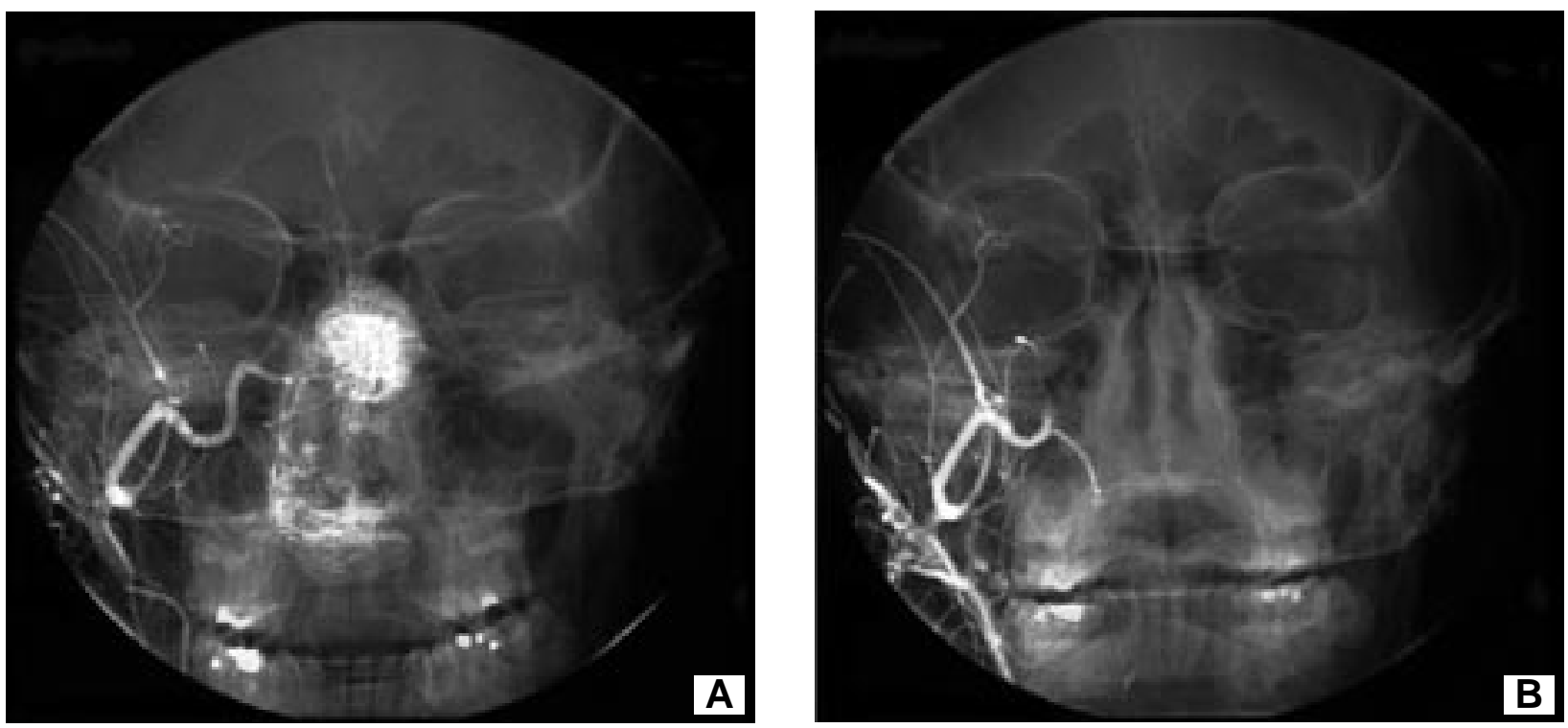

Figure 4. Pre-operative embolization of the angiofibroma using CT scan with subtraction technique and contrast (coronal views). A: Prior to embolization, a diagnostic vascular blush can be seen here. The vessel feeding the tumor is the internal maxillary artery. B: Post-embolization, the tumor's blood supply is severely limited and the classical vascular blush appearance is no longer evident.

complications were encountered in the patient described in the current report.

Embolization of feeding vessels is often performed prior to surgical resection of the tumor. This procedure is illustrated in Figure 4, where the tumor bulk is shown to have vanished after the blood supply has been interrupted by an embolus. In order to accomplish this, a catheter is inserted within the internal maxillary artery and Gelfoam pledgets suspended in contrast material are injected until blood flow is visibly reduced (19). This established procedure has been reported to reduce the otherwise severe blood loss. Only one discordant study has reported that embolization made no difference in blood loss and that the average blood loss for all 24 patients in that study was $1784 \mathrm{ml}$ (20). A more recent study reported seven patients having 10 procedures without embolization (mean blood loss 5380 $\mathrm{ml}$ ) and 13 patients having 16 procedures with embolization (mean blood loss $1037.5 \mathrm{ml}$ ), yet the difference in blood loss was only significant when comparing high grade tumors (21). A high standard deviation was reported in the non-embolized group, suggesting less vascularity in the low grade tumors. Perhaps, if only high grade tumors had been considered in the discordant 24 patient study, there would, in fact, be a difference between the embolized and nonembolized groups. In the current case of JNA, $1200 \mathrm{ml}$ of blood loss was estimated, which is consistent with the values stated in the studies using embolization. Another study with 43 cases reported that morbidity, recurrence and intraoperative complications decreased with embolization and skull based surgical approaches
(22), which further supports the use of embolization prior to surgery. Risks of embolizing are, however, numerous and include infections, bleeding, strokes, and allergic reactions. Again, the patient in this case was spared of any such complications.

External beam radiation is another treatment modality occasionally used alone or in conjunction with surgery. Wiatrak et al. reported three cases with extensive intracranial extension, all of which were treated with radiation therapy as a primary treatment (23). Symptoms were alleviated in patients and no serious side effects were noted, yet none had complete resolution, which makes radiotherapy seem suboptimal as a primary treatment. However, there are no better alternatives in cases with intracranial extension of the tumor. Another study of 13 JNA patients included two with intracranial involvement who received radiotherapy. These two patients did not have a recurrence (follow-up of 136 months average for all 11 subjects) (24). A third larger scale study seems even more optimistic, reporting 55 patients with JNA, 42 of which were treated with radiotherapy as a primary treatment, the rest having had radiation for recurrence post surgery. Most of the tumors in this series ( $80 \%$ after a minimum follow up of three years), were successfully treated (25). Conclusions from these studies suggest that radiotherapy be used in advanced lesions extending intracranially (unattainable by surgery), as well as in cases with multiple recurrences. However, radiation therapy should be limited to only certain cases since complications can be quite severe. Some complications include brain stem compromise, 
pituitary dysfunction, malignancies, xerostomia, caries, cataract formation, glaucoma and necrosis. This form of therapy was not considered for the current patient's management, as the tumor did not extend intracranially and it could be easily accessed by a transpalatal approach.

Hormonal therapy is a less favored treatment option and is no longer widely used, though it is still a major focus of research. Receptors for hormones such as progesterone, estradiol, testosterone and dihydrotestosterone have been detected in JNA tissue, but the results have been inconsistent between studies (26-28). Hormonal theories are very old and seem intuitive since JNA occurs almost exclusively in males and almost always in the adolescent years. Gates et al. reported success with hormones, having treated five patients with a testosterone receptor blocker (flutamide) for six weeks pre-operatively (29). The tumor size, measured by axial CT, was seen to decrease an average of $44 \%$ in four out of five tumors. A second non-clinical study showed that androgens seem to have no effect. This study designed tumor models for JNA, by transplanting the tumor into the subdermal space of athymic mice, and also by culturing it in vitro. The tumor failed to grow but did survive. Androgen treatment had no effect on survival or growth. The authors concluded that the growth of JNA requires factors other than androgens, or at least factors in addition to the androgens (30).

Some suggest that natural regression of JNA occurs, generally in the late teens or early twenties, though this has not been convincingly demonstrated. Most agree that there is some degree of shrinking, hardening and loss of vascularity occurring with age, and there are a few cases of regressions reported in the literature $(31,32)$. In reviewing these cases, it is most important to scrutinize the method and certainty of the diagnosis.

Recurrence is a common problem in JNA treatment. The rates post-surgery vary and depend on the adequacy of the approach, the conditions during the operation, the experience of the surgeon, as well as the extent of the lesion. The rate of recurrence is roughly $20 \%$ (6). A recent study by Radkowski et al. found that pre-operative tumor stage was the most important factor in determining the chances of recurrence after surgery (33). Other factors considered included the time of presentation, the intraoperative blood loss, and the surgical approach. These authors reported that five out of 23 cases $(21.7 \%)$ recurred after an average of six years follow-up. A second study reported similar figures of five recurrences out of $24(21 \%)$ (20), with an average follow-up period of 19 months. The recurrences are often treated with further surgery or radiotherapy. The overall patient survival of patients with JNA is 95\% (6). No recurrence eight months post-surgery has been observed in the patient presented in this case.

Malignant transformation is usually not a concern when dealing with JNA, but there have been some rare reports of fibrosarcoma transformation. This is thought to be associated with prior radiation therapy (6).

\section{CONCLUSION}

The diagnosis of JNA is based on a good history and physical exam, as well as imaging techniques and histology. On history, events of epistaxis unrelated to trauma should be identified, and other causes of epistaxis should be excluded. On rhinoscopy, a fleshy mass is most suggestive of JNA. The most useful imaging techniques for diagnosis are MRI, as well as arteriogram with contrast and subtraction technique. All of these investigations pointed to JNA in the current case report. Once the diagnosis is established, treatment planning is the next step.

Surgical resection is usually the preferred primary treatment for JNA and was judged to be most appropriate for the patient. Pre-operative embolization, which again was utilized, is often used to help control blood loss during surgery. Radiation therapy is generally reserved for recurrent lesions and extensive intracranial tumors and was not necessary in the current case. Hormonal therapy is sometimes, though rarely, used pre-operatively to reduce blood loss during surgery; this treatment modality was not considered in the case presented. The recurrence rate of JNA is relatively high and proper follow up is necessary for management. The patient discussed in this case report has had no recurrence and is examined on a regular basis. Natural regression is still controversial, and it is not suggested to refrain from treatment when JNA first presents. Malignant transformation, probably resulting from radiation therapy, has been reported, and for this reason it is important to restrict the use of external beam radiation to cases that cannot be treated with other methods mentioned in the text. In summary, JNA tumor treatment should be individualized for each case, depending on the extent, aggressiveness and presence of intracranial extension.

\section{REFERENCES}

1. Harrison DF. Juvenile postnasal angiofibroma - an evaluation. Clinical Otolaryngology 1: 187-97; 1976.

2. Martin H, Ehrlich HE, Abels JG. Juvenile nasopharyngeal angiofibroma. Annals of Surgery 127: 513-536; 1948.

3. Shaheen OH. Angiofibroma. In: Hibbert J, Kerr AG, eds. ScottBrown's Otolaryngology, Volume 5, 6th Edition. Bath, Great Britain: Reed Educational and Professional Publishing Ltd.; 1997: 1-6.

4. Neel HB III, Whicker JH, Devine KD, et al. Juvenile 
angiofibroma: review of 120 cases. American Journal of Surgery 126: 547-556; 1973.

5. Monsein LH. Interventional Neuroradiology of the Head and Neck. In: Krause CJ, Cummings CW, eds. Otolaryngology Head and Neck Surgery, Volume 1, 2nd Edition. St.Louis, Missouri: Mosby Year Book; 1986: 72-87.

6. Neville BW. Soft tissue tumors. In: Neville BW, Damm DD, Allen CM, et al., eds. Oral and Maxillofacial Pathology. Philadelphia, Pennsylvania: W.B. Saunders Company; 1995: 362-415.

7. Lee DA, Sessions DG. Juvenile nasopharyngeal angiofibroma. Surgical Rounds 3: 38; 1980.

8. Waldman SR, Levine HL, Astor F, et al. Surgical experience with nasopharyngeal angiofibroma. Archives of Otolaryngology 107: 677-682; 1981.

9. Sessions RB, Wills PI, Alford BR, et al. Juvenile nasopharyngeal angiofibroma: radiographic aspects. Laryngoscope 86: 2-18; 1976.

10. Lund VJ. Sinonasal pathology and the orbit. In: Mackay IS, Kerr AG, eds. Scott-Brown's Otolaryngology, Volume 4, 6th Edition. Bath, Great Britain: Reed Educational and Professional Publishing Ltd.; 1997: 1-19.

11. DeSanto LW. Neoplasms. In: Krause CJ, Cummings CW, eds. Otolaryngology-Head and Neck Surgery, Volume 1, 2nd Edition. Mosby-Year Book Inc., St-Louis, Missouri; 1993: 754764.

12 Hyams JV, Batsakis JG, Michaels L. Tumors of the Upper Respiratory Tract and Ear. In: Atlas of Tumor Pathology, Fascicle 25, Series 2. Washington, D.C.: Armed Forces Institute of Pathology; 1986: 130-134.

13. English GM, Hemenway WG, Cundy RL. Surgical treatment of invasive angiofibroma. Archives of Otolaryngology 96: 312318; 1972.

14. Jafek BW, Nahum AM, Butler RM, et al. Surgical treatment of juvenile nasopharyngeal angiofibroma. Laryngoscope 83: 707720; 1973.

15. Shaheen OH. Swellings of the infratemporal fossa. Journal of Laryngology and Otology 96: 817-836; 1982.

16. Howard DJ, Lund VJ. The midfacial degloving approach to sinonasal disease. Journal of Laryngology and Otology 106: 1059-1062; 1992.

17. Maharaj D, Fernandes CM. Surgical experience with juvenile nasopharyngeal angiofibroma. Annals of Otology, Rhinology and Laryngology 98: 269-272; 1989.

18. Jacobsson M, Petruson B, Svendsen P, et al. Juvenile nasopharyngeal angiofibroma. A report of eighteen cases. Acta Otolaryngologica (Stockholm) 105: 132-139; 1988.

19. Cotton RT, Ballard ET, Going JA, et al. Tumors of the head and neck in children. In: Thawley SE, Panje WR, Batsakis JG, et al., eds. Comprehensive Management of Head and Neck Tumors, Volume 2. Philadelphia, Pennsylvania: W.B.Saunders Company; 1987: 1770-1825.

20. da Costa DM, Franche GL, Gessinger RP, et al. Surgical experience with juvenile nasopharyngeal angiofibroma. Annales d'Oto-Laryngologie et de Chirurgie Cervico-Faciale 109: 231234; 1992.

21. Moulin G, Chagnaud C, Gras R, et al. Juvenile nasopharyngeal angiofibroma: comparison of blood loss during removal in embolized group versus nonembolized group. Cardiovascular and Interventional Radiology 1995;18:158-161.

22. Ungkanont $\mathrm{K}$, Byers RM, Weber RS, et al. Juvenile nasopharyngeal angiofibroma: an update of therapeutic management. Head and Neck 18: 60-66; 1996.

23. Wiatrak BJ, Koopmann CF, Turrisi AT. Radiation therapy as an alternative to surgery in the management of intracranial juvenile nasopharyngeal angiofibroma. International Journal of Pediatric Otorhinolaryngology 28: 51-61; 1993.

24. Fields JN, Halverson KJ, Devineni VR, et al. Juvenile nasopharyngeal angiofibroma: efficacy of radiation therapy. Radiology 176: 263-265; 1990.

25. Cummings BJ, Blend R, Keane $\mathrm{T}$, et al. Primary radiation therapy for juvenile nasopharyngeal angiofibroma. Laryngoscope 94: 1599-1605; 1984.

26. Kumagami $H$. Testosterone and estradiol in juvenile nasopharyngeal angiofibroma tissue. Acta Otolaryngologica (Stockholm) 111: 569-573; 1991.

27. Kumagami H. Sex hormones in juvenile nasopharyngeal angiofibroma tissue. Auris, Nasus, Larynx 20: 131-135; 1993.

28. Antonelli AR, Cappiello J, Di Lorenzo D, et al. Diagnosis, staging, and treatment of juvenile nasopharyngeal angiofibroma (JNA). Laryngoscope 97: 1319-1325; 1987.

29. Gates GA, Rice DH, Koopmann CF Jr, et al. Flutamide-induced regression of angiofibroma. Laryngoscope 102: 641-644; 1992.

30. Shikani AH, Richtsmeier WJ. Juvenile nasopharyngeal angiofibroma tumor models. Failure of androgens to stimulate growth in nude mice and in vitro. Archives of Otolaryngology Head and Neck Surgery 118: 256-9; 1992.

31. Weprin LS, Siemers PT. Spontaneous regression of juvenile nasopharyngeal angiofibroma. Archives of Otolaryngology Head and Neck Surgery 117: 796-799; 1991.

32. Jacobsson M, Petruson B, Ruth M, et al. Involution of juvenile nasopharyngeal angiofibroma with intracranial extension. A case report with computed tomographic assessment. Archives of Otolaryngology - Head and Neck Surgery 115: 238-239; 1989.

33. Radkowski D, McGill T, Healy GB, et al. Angiofibroma. Changes in staging and treatment. Archives of Otolaryngology Head and Neck Surgery 122: 122-129; 1996.

Johanna Choremis completed the Honours Health Science Program at Marianopolis College (CEGEP) (Montreal, Quebec, Canada) after which she was accepted into the five year program of medicine at McGill University (Montreal, Quebec, Canada). She has completed research in neuropharmacology at McGill. She is presently working on a research project concerning edema and steroids and has recently completed her third year in medical school. Saul Frenkiel, M.D., F.R.C.P.(C) received his M.D.C.M. from McGill University (Montreal, Quebec, Canada) in 1971. Following this, he completed postgraduate medical training in otolaryngology at the University of Toronto (Toronto, Ontario, Canada) in 1976, after which he received his F.R.C.S.(C) and his American Board certification. He is presently Chief of Otolaryngology at the Jewish General Hospital (Montreal, Quebec, Canada) and Program Director of Otolaryngology at McGill University. 